\title{
Heat Shock Protein B8 (HSPB8) Reduces Oxygen-Glucose Deprivation/Reperfusion Injury via the Induction of Mitophagy
}

\author{
Fazhao Lia Jieqiong Tan ${ }^{b}$ Fangfang Zhouc Zhiping Huc Binbin Yang ${ }^{c}$ \\ aDepartment of General Surgery, 2nd Xiangya Hospital, Central South University, Changsha, bNational \\ Key Laboratory of Medical Genetics, Central South University, Changsha, 'Department of Neurology, \\ 2nd Xiangya Hospital, Central South University, Changsha, China
}

\section{Key Words}

Cerebral • Ischemia/Reperfusion • HSPB8 • Mitophagy • Neuroprotection

\begin{abstract}
Background/Aims: We have reported the neuroprotective properties of Heat shock protein B8(HSPB8) against oxygen-glucose deprivation/reoxygenation (OGD/R)-induced injury by inhibiting the mitochondrial apoptotic pathway. However, the exact underlying mechanism of its protective effect on mitochondrial function remains unknown. Here we examined whether the beneficial effect of HSPB8 on OGD/R-induced cell death is associated with mitophagy in mouse neuroblastoma Neuro2a (N2a) cells. Methods: Using the mouse transient middle cerebral artery occlusion (tMCAO) model and mouse neuroblastoma Neuro2a (N2a) cell cultures subjected to OGD/R, we employed western-blot, RT-PCR and immunostaining to analyze the change of expression pattern of HSPB8 and mitophagic flux after brain I/R both in vivo and in vitro. Moreover, via overexpressing HSPB8 or knocking down HSPB8 expression with siRNA in N2a cell, we evaluated the effect of HSPB8 on mitochondrial function during OGD/R. The impact of HSPB8 on mitophagic pathway was also assessed. Finally, mitotophagy inhibitors (CQ and Mdivi-1) were adopted to verify the involvement of mitophagy in HSPB8induced neuroprotection. Results: HSPB8 could be up-regulated by brain I/R both in vivo and in vitro. Mitophagy enhancement coincided with induction of HSPB8 during I/R. Overexpression of HSPB8 reinforced I/R-induced mitophagy in OGD/R-treated mouse N2a cells and HSPB8 silence suppressed mitophagy process. Inhibition of mitophagy compromised neuroprotection conferred by HSPB8 overexpression. Conclusions: HSPB8 promoted OGD/Rinduced mitophagy, which restored the mitochondrial function and contributed to the decrease in cell apoptosis after OGD/R. Therefore, HSPB8 could be a favorable neuroprotective agent for cerebral I/R related disorders.




\section{Cellular Physiology Cell Physiol Biochem 2018;48:1492-1504 and Biochemistry Published online: July 31, $2018 \quad \begin{aligned} & \text { DOI: 10.1159/000492259 } 2018 \text { The Author(s). Published by S. Karger AG, Basel } \\ & \text { www.karger.com/cpb }\end{aligned}$}

Li et al.: Neuroprotective Role of HSPB8 Induced Mitophagy in OGD/R

\section{Introduction}

As a member of the sHSPs (small heat shock proteins) family, HSPB8 is of particular interest in the field of neurological diseases since mutations in its DNA sequence correlate with the development of distal hereditary motor neuropathy and Charcot-Marie-Tooth disease. Moreover, it has been reported that HSPB8 could protect against intracellular accumulation of insoluble aggregates in various neurodegenerative diseases [1]. In addition, HSPB8 is upregulated in different models of myocardial ischemia [2,3] , and promotes cell survival in a context of acute, repetitive, and chronic ischemia $[4,5]$. Therefore, it is reasonable to speculate that HSPB8 could play a protective role in brain ischemia. Our previous study demonstrated that OGD/R upregulated HSPB8 expression and that HSPB8 overexpression ameliorated OGD/R injury-induced mouse N2a cell death, at least partially, through interfering with mitochondria-dependent apoptosis pathway [6]. In an ischemic animal model, HSPB8 reportedly prevented oxidative stress-induced mitochondrial dysfunction [7]. These studies highlight the possibility that HSPB8 targets mitochondria to protect the brain from ischemic injury. Mitochondrial dysfunction is well-established in cerebral ischemia. Mitochondria play key roles in various cell processes including ATP production, Ca2+ homeostasis, ROS generation and apoptosis. Neurons are particularly vulnerable to ischemia because of their high energy demands. It seems reasonable to hypothesize that neuronal resistance to ischemia is dependent on the ability of neurons to maintain mitochondrial homeostasis such as timely clearing dysfunctional mitochondria.

Interacting with Bag3, HSPB8 has been demonstrated to be involved in the delivery of misfolded proteins to the macroautophagy machinery, $[1,8,9]$. However, whether HSPB8 is directly involved in the mitophagy process remains unclear. In the present study, we first assessed the HSPB8 expression and mitophagy involvement in the mouse model of MCAO/R and in mouse N2a cells at different reperfusion time points after 4h OGD injury, then we employed in vitro assays to test the hypothesis that mitochondria are the targets of HSPB8mediated autophagy and that HSPB8 is protective through enhancing OGD/R-elicited mitophagy.

\section{Materials and Methods}

\section{Animals}

All the animals used in this study were pathogen-free male adult C57BL/6 mice weighing 22-25 g, provided by the Animal Center of 2nd Xiangya Hospital, Central South University. All mice had access to food and wate

$\mathrm{r}$ and kept in an air-conditioned room with a constant temperature of $21-22^{\circ} \mathrm{C}$. They were kept under a 12-hour light/dark cycle in separated clean cages. All feeding and breeding operations used in this study were compliant with the National Institutes of Health (NIH) Guide for the Care and Use of Laboratory Animals guidelines, which have also been approved by the Ethics Committee for Use of Experimental Animals in Central South University.

Transient middle cerebral artery occlusion (tMCAO)

Mice were anesthetized with ketamine/xylazine $(100 \mathrm{mg} / 10 \mathrm{mg} / \mathrm{kg})$ intraperitoneally. Body temperature was maintained at $37 \pm 0.5^{\circ} \mathrm{C}$ using a heating pad (RWD Life Science, Shenzhen, China). Animal surgery was performed as previously described [10]. Briefly, a 4-0 suture (Covidien, Mansfield, MA) with a round tip and silicon coating was inserted from the left external carotid artery (ECA) into the internal carotid artery (ICA). The suture reached the circle of Willis to occlude the origin of the middle cerebral artery (MCA). The success of occlusion was determined by monitoring the decrease in surface cerebral blood flow (CBF) to $20 \%$ of baseline CBF using a laser Doppler flow meter (Moor Instruments, Devon, UK). After 60 minutes, the nylon thread was then removed to allow reperfusion. All animals were allowed to 


\section{Cellular Physiology Cell Physiol Biochem 2018;48:1492-1504 \begin{tabular}{ll|l} 
and Biochemistry Published online: July 31, 2018 & $\begin{array}{l}\text { C } 2018 \text { The Author(s). Published by S. Karger AG, Basel } \\
\text { www.karger.com/cpb }\end{array}$
\end{tabular}}

Li et al.: Neuroprotective Role of HSPB8 Induced Mitophagy in OGD/R

recover from anesthesia and were sacrificed $1 \mathrm{~h}, 12 \mathrm{~h}, 24 \mathrm{~h}$ after reperfusion respectively, for histological analysis and western-blot.

\section{Immunohistochemistry staining}

The $25-\mu \mathrm{m}$ brain slices were incubated with citrate buffer $(\mathrm{pH} 6.0)$ at $100{ }^{\circ} \mathrm{C}$ for $10 \mathrm{~min}$, and were blocked in the TBS solution containing 3\% BSA and $0.2 \%$ TX-100 for $1 \mathrm{~h}$ at room temperature. Then, they were incubated with HSPB8 primary antibody $\left(1: 50\right.$, Abcam, ab151552) at $4{ }^{\circ} \mathrm{C}$ overnight. After washes with TBS solution containing $0.1 \%$ TX-100, the antibody-labeled brain slices were incubated with $1 \%$ BSA containing fluorescence labeled anti-rabbit IgG $(1: 200)$ for $1 \mathrm{~h}$ at room temperature. Sections were rinsed in TBS, mounted onto slides, air-dried, cleared in xylene, and coverslipped in DPX (Sigma).

\section{Cell culture and $O G D / R$ process}

Mouse neuroblastoma Neuro2a (N2a) cells were purchased from ATCC. The method of mouse N2a cell culture preparation has been described in detail previously. OGD was performed as described [11]. The high-glucose DMEM culture medium supplemented with 10\% FBS was replaced with Earle's balanced salt solution (Leagene Biotech Co. Beijing, China), and then cells were immediately moved into an airtight hypoxic incubator (MGC AnaeroPack®-Anaero Mitsubishi Gas Chemical Co., Inc. Japan). The incubator was kept in $37^{\circ} \mathrm{C}$ for $4 \mathrm{~h}$ to initiate the OGD insult. After that, OGD was terminated by replacing Earle's balanced salt solution with high-glucose DMEM medium supplemented with $10 \% \mathrm{FBS}$, and the cells were further incubated for an additional $0,4,12 \mathrm{~h}$ under normoxic conditions at $37^{\circ} \mathrm{C}$ respectively. Inhibitors, $10 \mu \mathrm{mol} / \mathrm{L}$ CQ (Sigma, C6628) or $1.25 \mathrm{mmol} / \mathrm{L}$ 3-MA (Sigma, M9821), were applied at the onset of reperfusion.

\section{Transfection and RNA silencing in cultured mouse N2a cells}

A vector containing mice HSPB8-Myc cDNA was obtained from ATCC. Mouse N2a cells were cultured for 96 hours and then transfected with the indicated plasmids using Lipofectamine 2000 (Invitrogen) and Lipofectamine LTX with PLUS Reagent (Invitrogen) according to manufacturer's instructions. siRNA for HSPB8 (targeting sequence: 5'-AGAGCAGUUUCAACAACGA) and a scrambled control siRNA (5'-AUGAAGTGAAUUGCUCAA-3') were synthesized by GenePharm(Shanghai). mouse N2a cells were transfected on $5 \mathrm{~d}$ in vitro with $20 \mathrm{nmol}$ HSPB8siRNA or scrambled siRNA using Lipofectamine RNAiMAX (Invitrogen, 13778100). After transfection in antibiotic-free medium for $8 \mathrm{~h}$, cells were refreshed with normal medium.

\section{Confocal microscopy}

For microscopy examination, mouse N2a cells were fixed with $4 \% \mathrm{PFA} / \mathrm{PBS}$ solution for $15 \mathrm{~min}$ at $25^{\circ} \mathrm{C}$, then permeabilized by $0.5 \%$ Triton X-100/PBS solution for $5 \mathrm{~min}$ at $25^{\circ} \mathrm{C}$. After blocking of samples with $1 \%$ BSA/PBS solution for 30 min with gentle shaking, and incubated with LC3 antibody (1:25; Sigma, L7543) and anti-TOMM20 antibody (1:250, Abcam, ab186734) in 1\% NGS/PBS at room temperature for 1 hour. Fluorescent dye-conjugated secondary antibody: anti-mouse alexa Fluor 488(1:500, Abcam, ab150117) and anti-rabbit Alexa Fluor 594(1:500, Abcam ab150152) were added and incubated at room temperature for 30 minutes. Dishes were observed on a confocal microscope (Fluoview FV1000, Olympus, Tokyo, Japan). Images were analyzed by Image Pro-Plus 7.0 software.

\section{Real-time PCR}

Total RNA was isolated from lysed cells using Trizol (Sigma, T9424) and purified according to manufacturer's instructions. Reverse transcription was performed by using the Reverse Transcription Kit (Promega). Equal amounts of total RNA (500 ng) were reverse-transcribed. The following primer sequences were used:HSPB8 (Fw: 5'-CCGGAAGAACTGATGGTAAAGAC-3', Rev: 5' - CCTCTGGAGAAAGTGAGGCAAATAC-3'), TOMM20(Fw: 5' TGGGCTTTCCAAGTTACCTGATT-3'; Rev: 5'- CACCCTTCTCGTAGTCACCTTGT-3'), Cox411 (Fw: 5'-GAATGTTGGCTTCCAGAGCG-3'; Rev: 5'-TCACAACACTCCCATGTGCT-3'). The PCR experiments were repeated 3 times, each using separate sets of cultures.

Western blot analysis

Cultured cells were lysed in Laemmli SDS sample buffer directly, and brain homogenates were diluted in $2 \times$ Laemmli SDS sample buffer at 1:1 ratio, followed by heating at $95^{\circ} \mathrm{C}$ for 5 minutes. Samples were 


\section{Cellular Physiology Cell Physiol Biochem 2018;48:1492-1504 and Biochemistry Published online:July 31, $2018 \quad \begin{aligned} & \text { DOI: 10.1159/000492259 } 2018 \text { The Author(s). Published by S. Karger AG, Basel } \\ & \text { www.karger.com/cpb }\end{aligned}$}

Li et al.: Neuroprotective Role of HSPB8 Induced Mitophagy in OGD/R

subjected to 10-12\% SDS-PAGE and transferred onto nitrocellulose membrane. Membranes were probed with primary antibodies, including anti-LC3 (1:1000; Sigma, L7543), anti-COX4l1 (1:1000, CST, 4844S) and mouse anti-TOMM20 (1:1000, Abcam, ab186734). Anti- HSPB8(1:1000, CST, 3059S), anti-caspase9 (1:500, Abcam, ab69514), anti-caspase 8 (1:1000, Abcam, ab25901) and anti-caspase 3 (1:200; Abcam, ab4051) for 1-2 hours at room temperature. After extensive washing, the membrane was incubated with appropriate secondary antibodies (1:1000). Immunoreactive bands were visualized by the ECL substrate (Pierce). The chemiluminescence level was recorded using an imaging system (Bio-Rad, Hercules, CA). The results were normalized to loading control $\beta$-actin.

\section{Flow Cytometric analysis}

Apoptosis was detected by Annexin V FITC Apoptosis Detection Kit (Sigma). Briefly, N2a cells were collected and washed twice with PBS. $500 \mu \mathrm{L}$ binding buffer suspension was then added to the treated cells. After that, $5 \mu \mathrm{L}$ Annexin V-FITC and $10 \mu \mathrm{L}$ propidium iodide were added to each group and cultures were incubated at $37^{\circ} \mathrm{C}$ for $5 \sim 15 \mathrm{~min}$ in dark. Flow cytometer (BD, Biosciences) was used to detect the percent cells with apoptosis and flowJo software was used for flow cytometry analysis.

\section{The terminal deoxynucleotidyl transferase-mediated dUTPbiotin nick end labeling (TUNEL) assay}

Cells were previously seeded on poly-l-lysine-treated coverslips. After treatment, fixed cells were stained using a TUNEL kit according to the manufacturer's instructions (Click-iT Plus TUNEL Kit, Thermo Fisher), and cell nucleuses were stained with DAPI. For each coverslip, 5 random fields were examined under a fluorescent confocal microscope. The total cell number was counted using DAPI staining, and the average TUNEL-positive cell ratio was calculated.

\section{Electron microscopy}

Cells was fixed with $4 \%$ glutaraldehyde in $0.1 \mathrm{~N}$ sodium-cacodylate at room temperature for $1 \mathrm{~h}$, and processed for electron microscopy using a standard protocol [12]. Cells were fixed with $2 \%$ glutaraldehyde in 0.2M 4-(2-Hydroxyethyl) piperazine-1-ethanesulfonic acid sodium salt (HEPES) (pH 7.4) for $2 \mathrm{~h}$ at room temperature. Fixed cells were collected and postfixed with $1 \% 0$ s04 and stained in 2\% uranyl acetate (Eskelinen, 2008). The pellets were dehydrated through a graduated ethanol series and embedded in Epon 812 (Electron Microscopic Sciences) for sectioning and observed under a transmission electron microscope (Hitachi H7500). A series of ultrastructural images were collected with a bottom-mount digital camera for the analysis of autophagosomes.

\section{Cytochrome c Oxidase (COX) Activity Assays}

Mitochondria of N2a cells were obtained using a mitochondria isolation kit (Genmed Scientifics, Cat. GSM10006). Mitochondria were suspended in $50 \mu \mathrm{L}$ of isolation buffer containing $250 \mathrm{mM}$ sucrose, $20 \mathrm{mM}$ HEPES, pH 7.2, and $1 \mathrm{mM}$ EDTA. Suspensions were added to a cuvette containing $0.95 \mathrm{~mL}$ of $1 \mathrm{x}$ assay buffer (10 mM Tris- $\mathrm{HCl}, \mathrm{pH} 7.0$, and $120 \mathrm{mM} \mathrm{KCl}$ ), and the reaction volume was brought to $1.05 \mathrm{~mL}$ with $1 \mathrm{x}$ enzyme dilution buffer (10 mM Tris-HCl, pH 7.0). Cytochrome c oxidase activity then was measured using cytochrome c oxidase assay kit (Sciencell, Cat. 8278). In brief, Ferrocytochrome $c$ (reduced cytochrome $c$ with dithiothreitol) was then added to the sample, and COX activity was measured by the decrease in absorption at $550 \mathrm{~nm}$. The reading was recorded every 5 seconds during the first 3 minutes. Background levels were measured without cell suspensions. All measurements were repeated at least three times. All normalized control of cytochrome c oxidase activity values will be 1 (or 100\%).

\section{Determination of Mitochondrial ATP Synthase Activity}

ATP synthase activity was analyzed using the ATP synthase microplate kit (Genmed Scientifics), following the manufacturer's protocols. Briefly, mitochondrial membrane proteins were isolated and immunocaptured in the wells of the microplate. The ATP synthase activity was measured by monitoring the conversion of ATP to ADP by ATP synthase coupled to the oxidation reaction of NADH to NAD+ with a 


\section{Cellular Physiology Cell Physiol Biochem 2018;48:1492-1504 and Biochemistry Published online: July 31, $2018 \quad \begin{aligned} & \text { DOI: 10.1159/000492259 } 2018 \text { The Author(s). Published by S. Karger AG, Basel } \\ & \text { www.karger.com/cpb }\end{aligned}$}

Li et al.: Neuroprotective Role of HSPB8 Induced Mitophagy in OGD/R

reduction in absorbance at 340nm All measurements were repeated at least three times. All normalized control of complex $\mathrm{V}$ activities values will be 1 (or $100 \%$ ).

\section{Statistical analysis}

Data were expressed as the mean \pm standard error of the mean (SEM). Student's $t$ test was used to determine the difference between two experimental groups. One-way analysis of variance (ANOVA) and Dunnett's post hoc test was used for multiple comparisons between more than two groups. P values $<0.05$ were considered statistically significant.

\section{Results}

HSPB8 is upregulated by brain ischemia

To determine whether the expression of HSPB8 was affected by brain ischemia, mice with transient middle cerebral artery occlusion (tMCAO) and cultured mouse N2a cells exposed to OGD/R were used as in vivo and in vitro models of cerebral ischemia, respectively. Western blot and immunohistochemistry showed low levels of HSPB8 protein in CA1 sector of the hippocampus before treatment. $1 \mathrm{~h}$ MCAO followed by reperfusion led to a time-dependent elevation of HSPB8 (Fig. 1A-1C). Correspondingly, in mouse N2a cells, western blot analysis clearly indicated that OGD/R induced HSPB8 expression in a time-dependent pattern from $4 \mathrm{~h}$ reperfusion after $4 \mathrm{~h}$ OGD and the difference became most pronounced at $12 \mathrm{~h}$ perfusion (Fig. 1D-1E). RT-PCR analysis also showed that OGD/R dramatically increased HSPB8 at the mRNA level. The gene expression of HSPB8 was significantly upregulated immediately after $4 \mathrm{~h}$ OGD and the peak was observed at $12 \mathrm{~h}$ reperfusion after OGD (Fig. $1 F)$. When compared with that in 0h reperfusion, HSPB8 mRNA expressions were significantly higher in $4 \mathrm{~h}$ and $12 \mathrm{~h}$ reperfusion post-OGD. Taken together, brain ischemia promoted the transcription and translation of HSPB8 in our mouse and N2a cell model.

\section{Brain ischemia-reperfusion induced mitophagy}

In the present study, a time course analysis of protein expressions including autophagic markers LC3(suggesting the activation of autophagy) and p62/SQSTM1(an autophagy substrate responsible for delivering ubiquitinated proteins for degradation, which is itself degraded by autophagy) was conducted in the ipsilateral hemisphere at 1, 12 and 24, and

Fig. 1. Brain ischemia/reperfusion upregulates HSPB8 in vivo and in vitro. A HSPB8 immunohistochemistry in the hippocampus of the sham (a, e) $1 \mathrm{~h}(\mathrm{~b}, \mathrm{f}) 12 \mathrm{~h}(\mathrm{c}, \mathrm{g}) 24 \mathrm{~h}(\mathrm{~d}, \mathrm{~h})$ of reperfusion after $1 \mathrm{~h}$ MACO. HSPB8 was visualized using 3, 3'-diaminobenzidine tetrahydrochloride, hematoxylin used as a counterstain. Scale bar $=200$ $\mu \mathrm{m}$ (a-d), $100 \mu \mathrm{m}$ (e-h). Images e, f, g and h were at higher magnifications from $\mathrm{a}, \mathrm{b}, \mathrm{c}$ and $\mathrm{d}$ respectively. B HSPB8 protein levels were determined by western blot analysis at different time points after the onset of reperfusion in $1 \mathrm{~h}$ MCAO mice. $\beta$-actin was used as the internal control. C The statistical analysis of HSPB8 protein level. D and E

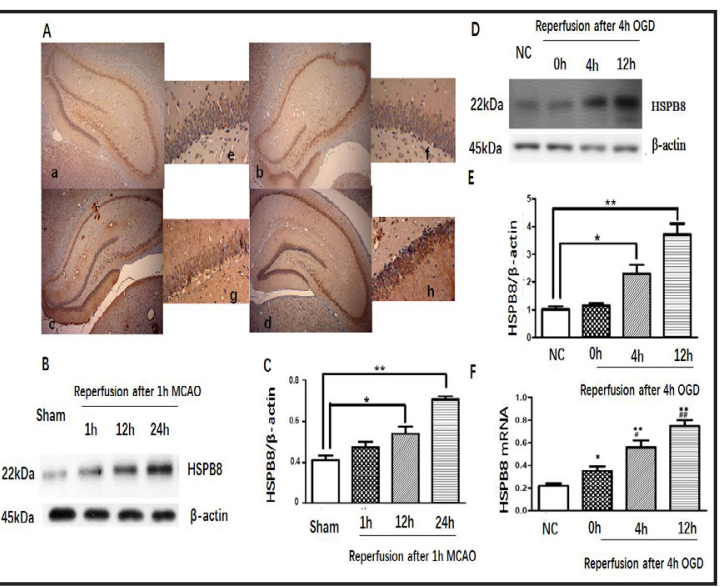
HSPB8 protein levels were determined by western blot analysis at $0 \mathrm{~h}, 4 \mathrm{~h}, 12 \mathrm{~h}$ of reperfusion after $4 \mathrm{~h}$ OGD in mouse N2a cells. E HSPB8 protein levels were quantified according to the results of three independent blots. F HSPB8 mRNA expression levels in cultured mouse N2a cells after OGD/R. Actin mRNA was used as an internal control. Experiments were repeated three times. Data are presented as the mean \pm SEM. $* \mathrm{p}<0.05, * * \mathrm{p}<0.01$ compared with sham/NC group. \# $\mathrm{p}<0.05$, \#\#p<0.01 compared with 0 h reperfusion group.

\section{KARGER}


$48 \mathrm{~h}$ reperfusion post-ischemia. As western blot showed, the turnover of LC3-II/LC3-I increased as early as $1 \mathrm{~h}$ after reperfusion. The transformation was significant at $12 \mathrm{~h}$ and peaked at $24 \mathrm{~h}$ reperfusion after MCAO. Accordingly, the p62 levels decreased since $1 \mathrm{~h}$ after reperfusion and lasted up to $24 \mathrm{~h}$. The significant reduction started at $12 \mathrm{~h}$ with the minimum at $24 \mathrm{~h}$ reperfusion (Fig. 2A-2C). Meanwhile, the number of mitochondria was assessed by the constitutively-expressed COX411 and TOMM20 (mitochondrial outer membrane proteins indicating the amount of mitochondria). We observed a time-dependent decrease of the mitochondrial markers (Fig. 2A, 2C-D). The activation of autophagy and reduction of mitochondrial mass implicated mitophagy activation after MCAO/R.

\section{HSPB8 increased the induction of mitophagy}

During the above study, we noted mitophagy enhancement coincided with the induction of HSPB8, which implied that mitophagy pathway may be involved in HSPB8's action. We then test if HSPB8 may be regulating mitophagy after brain ischemia/reperfusion using an in vitro OGD/R model. The N2a cultures transfected with a HSPB8 or a vehicle vector were subjected to $4 \mathrm{~h}$ OGD followed by $12 \mathrm{~h}$ reperfusion. Western blotting analysis confirmed

Fig. 2. Mitophagy induction after cerebral ischemia/reperfusion. Mice were subjected to $1 \mathrm{~h}$ MCAO followed by 1,12 and $24 \mathrm{~h}$ reperfusion. Extracts from the ipsilateral hemisphere were havested for immunoblotting. A Expression changes of LC3, p62 and mitochondrial markers including TOMM20 and COX411 at different times of reperfusion was determined by western blot. Levels of $\beta$-actin protein were used as the loading control. B-E Bar charts showed the averages of protein levels/ $\beta$-actin by band density analysis. Experiments were repeated three times.Data are presented as the mean \pm SEM. ${ }^{* *} \mathrm{p}<0.01$ vs.

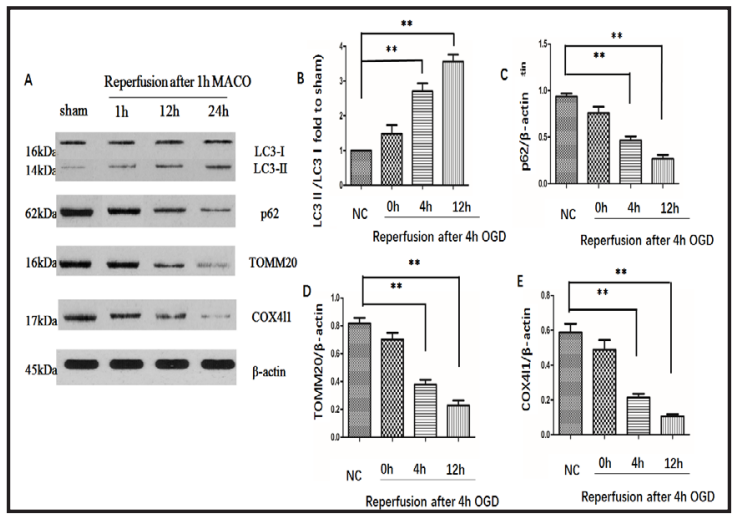
indicated group.

Fig. 3. HSPB8 enhanced mitophagy in $\mathrm{OGD} / \mathrm{R}$ treated mouse $\mathrm{N} 2 \mathrm{a}$ cells. N2a cells were subjected to $4 \mathrm{~h}$ OGD and then treated by $12 \mathrm{~h}$ reperfusion. A HSPB8 protein levels were determined by western blot analysis in N2a cells transfected with control or HSPB8 vector. B Cells were assessed for LC3 production, p62 expression and levels of TOMM20 and COX4l1 in seperated groups by western blot analysis. C-E Protein levels were quantified based on the results of three independent blots. F. Cox4l1 and Tomm20 mRNA levels were determined by quantitative RT-PCR and were normalized relative to the control. Experiments were repeated three times. Data represent the mean \pm SEM. ${ }^{*} \mathrm{p}<0.05,{ }^{* *} \mathrm{p}<0.01$ compared with the indicated group.

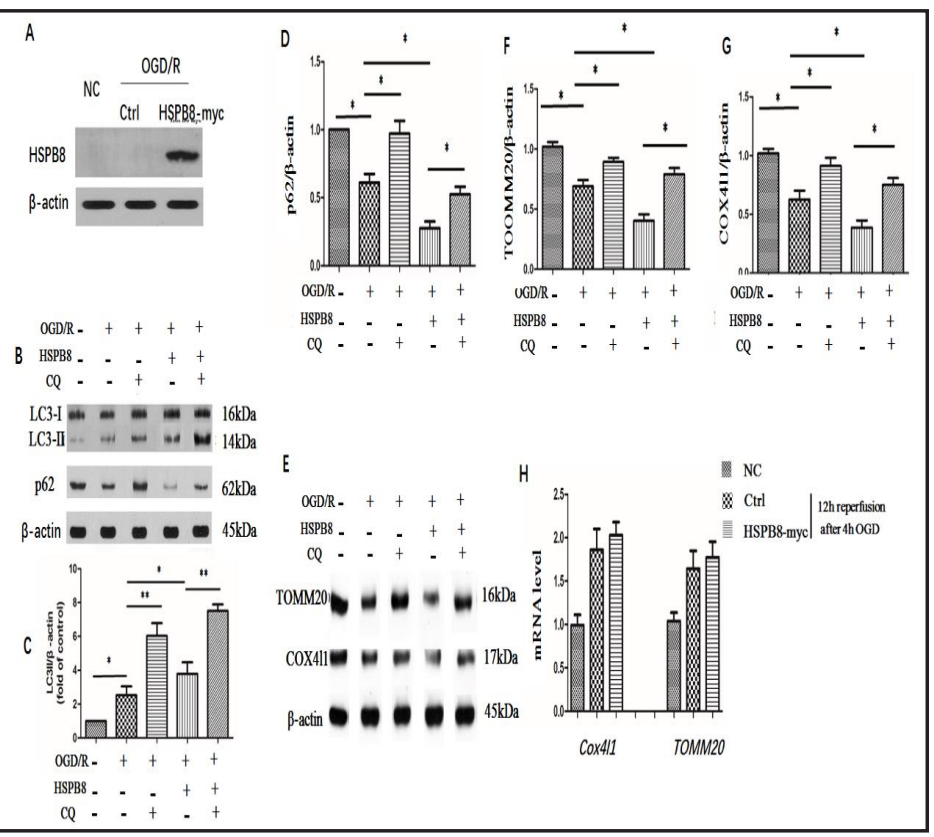


that transfection of HSPB8 resulted in overexpression of HSPB8 in N2a cells (Fig. 3A). The conversion from LC3-I to LC3-II in N2a cells increased and the level of p62 decreased after OGD/R compared with NC group. HSPB8 overexpression augmented autophagy after OGD/R as demonstrated by a statistically significant increased LC3 flux and reduced p62 expression when compared with that in cells transfected with vehicle vector (Fig. 3B-3D). Furthermore, after OGD/R, Cox4l1 and TOMM20 protein levels were reduced to a greater extent in HSPB8 overexpressing cells compared with that in cells transfected with vehicle vector (the difference was statistically significant) (Fig. 3B, 3E). This loss of mitochondrial mass may be due to mitochondria degradation, or alternatively caused by decreased mitochondrial biogenesis. We thus determined the mRNA levels of COX4 and TOMM20 by real-time PCR. As shown in Fig. 3F, the genes of the mitochondrial markers were not significantly downregulated by HSPB8 overexpression. These data suggested that HSPB8 reduced mitochondria mass by facilitating mitochondrial degradation rather than reducing the transcription.

To explore that HSPB8 mediated mitochondrial clearance was autophagic, the lysosome inhibitor chloroquine(CQ) was employed. According to the results shown in Fig. 3B-3D, upregulation of LC3B-II by HSPB8 was potentiated by CQ and reduction of p62 level by HSPB8 overexpression was inhibited by CQ treatment, indicating the defective autophagic flux. Furthermore, CQ incubation significantly attenuated the reduction of TOMM20 and COX411 induced by HSPB8 overexpression (Fig. 3B, 3F), which suggested that autophagic mitochondria lysis occurred in HSPB8 overexpressing cells subjected to OGD/R.

To further confirm that mitophagy was enhanced by HSPB8 overexpression, mitochondria were stained with antibody against TOMM20 and autophagosomes were visualized with antibody against LC3. LC3 staining was hardly detectable in NC cells with mitochondria. After OGD/R, mitochondria with LC3 puncta substantially increased in cells transfected control vector compared with NC group, and significantly more LC3 puncta associated with mitochondria were observed in HSPB8 overexpressing cells (Fig. 4A-4B). Collectively, these data indicated that OGD/R-induced mitophagy can be further augmented by HSPB8 overexpression.

Fig. 4. HSPB8 overexpression augmented mitophagy in mouse N2a cell after OGD/R injury. A LC3 and TOMM20 double staining for the determination of mitophagy. Images were captured by confocal microscopy. Scale bar, $10 \mu \mathrm{m}$. B columns represent number of LC3 puncta associated with mitochondria. Experiments were repeated three times. Data represent the mean \pm SEM. $* \mathrm{p}<0.05$, ** $\mathrm{p}<0.01$ compared with the indicated group.

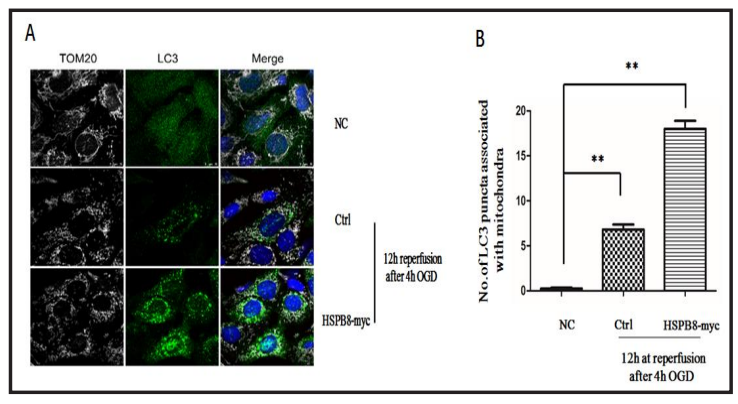

Fig. 5. RNA interference of HSPB8 suppressed the induction of Mitophagy. Mouse N2a cells were subjected to $4 \mathrm{~h}$ OGD follwed by $12 \mathrm{~h}$ reperfusion. A The ratio of LC3II/I was analyzed by Western analysis with anti-LC3 antibody. B Quantitative data of HSPB8 protein band intensity after normalization to $\beta$-actin. C. The representative immunoblots depicting the protein expression levels of COX4I1 and TOMM20 in separated groups. D-E Bar graphs represent the densitometry analysis of immunoblots normalized to the respective $\beta$-actin. Experiments were repeated three times. Data represent the mean \pm SEM. $* \mathrm{p}<0.05, * *$ $\mathrm{p}<0.01$ compared with the indicated group.

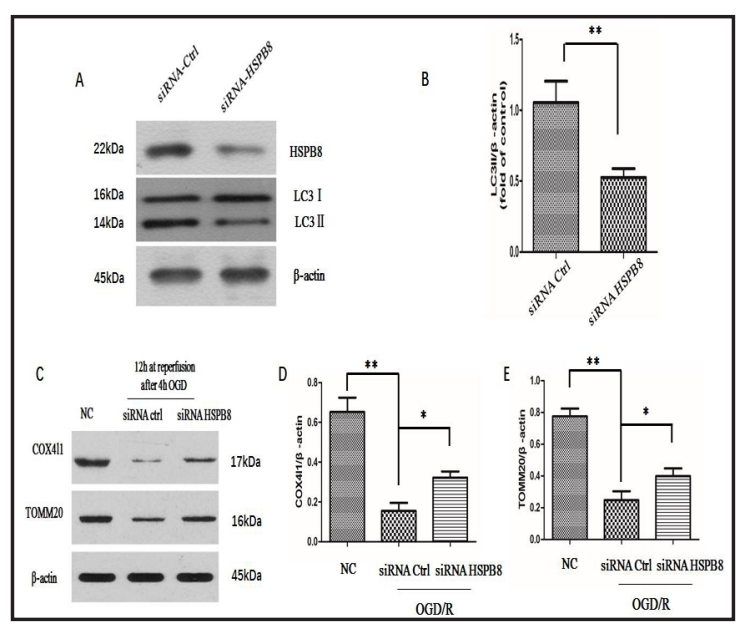


siRNA-HSPB8 suppressed the induction of mitophagy

To further explore the effect of HSPB8 on mitophagy, we transfected N2a cells with a specific siRNA against HSPB8. Knockdown of HSPB8 was assessed by western blot after 72 hours transfection. As shown in Fig. 5A, transfection with HSPB8 siRNA, but not control siRNA, reduced HSPB8 expression (50\% reduction in HSPB8). After 4h OGD followed by $12 \mathrm{~h}$ reperfusion, HSPB8 siRNA markedly diminished LC3 II/ I ratio compared with that in cells transfected with control siRNA (Fig. 5A-5B). The expressions of mitochondrial proteins COX4l1 and TOMM20 were increased in HSPB8 silent cells compared with that in cells transfected by control vector (Fig. 5C-5E). Moreover, a significant decrease in the colocalization of LC3 puncta with TOMM20 was also observed in the cells transfected with HSPB8 siRNA compared with that in the cells transfected with control siRNA (Fig. 6A$6 B$ ). Based on TEM, under OGD/R stress, in cells transfected with control siRNA, some of mitochondria appeared being encapsulated by double membranes of the autophagosomes. However, in the cells transfected with HSPB8 siRNAs, lack of mitophagy was observed (Fig. 6C-6D). Taken together, these data suggested that knockdown of HSPB8 resulted in suppressed mitophagy.

\section{Overexpression of HSPB8 attenuated OGD/R-induced apoptosis and mitochondria} dysfunction

The expression of HSPB8 was significantly increased during OGD/R, thus indicating a possible involvement of this protein in cell survival. It is well known that upregulation of sHSPs in response to cellular stress is one mechanism to increase cell viability. Many studies have described the cytoprotective effect of HSPB8 in motor neuron diseases [13], as well as during cardiac ischemia/reperfusion injury [5]. Therefore, we speculated that overexpression of HSPB8 in N2a cells may be a pro-survival factor in OGD/R setting. First, to investigate whether HSPB8 played a role in neuronal susceptibility to OGD/R stress, apoptosis was evaluated by the protein level of caspase 3 and flow cytometric analysis. The results indicated that after OGD/R, HSPB8 overexpression lowered caspase-3 expression compared with controlvector group (Fig. 7A-7B). In addition, N2a cells overexpressing HSPB8 exhibited less apoptosis as shown by a statistically significant decrease in the percentage of $\mathrm{AV}+\mathrm{PI}+$ cells than cells transfected control vector (Fig. 8A-8B). These data clearly indicated that HSPB8 overexpression rendered cells more resistant to OGD/R. To further investigate the role of HSPB8 in apoptosis, we examined the expressions of caspase-8 and caspase- 9 in each group. The data showed that there was a significant decrease in caspase- 9 expression in HSPB8 overexpressing cells but no significant changes in caspase-8 compared with control vector group after OGD/R, which implies that HSPB8 had an effect on mitochondria-related apoptosis but not on death receptor-mediated apoptosis (Fig. $7 A-7 B$ ). We further measured

Fig. 6. HSPB8 konck-down decreased autophagic removal of mitochondria. A LC3 and TOMM20 double staining for the determination of mitophagy with siRNA HSPB8 or scrambled siRNA. Scale bars $10 \mu \mathrm{m}$. Areas in the white boxes are enlarged on the right. D Panel columns represent number of LC3 puncta associated with mitochondria. F TEM-based determination of mitophagy in mouse N2a cells. The arrow indicates a mitochondrion encapsulated by the double membrane of an autophagosome. The typical structure indicating

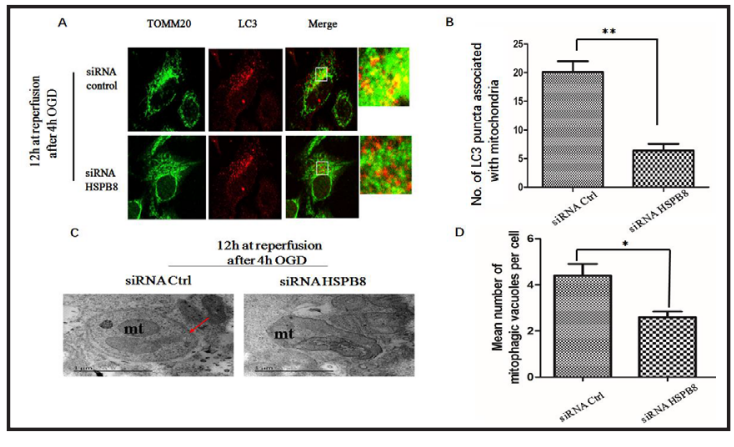
mitochondria undergoing mitophagy verified the effect of HSPB8 on mitophagy after OGD/R injury. D. Quantification of the average number of the mitophagic vacuoles per cell. Experiments were repeated three times.Data represent the mean \pm SEM. $*$ p $<0.05, * *$ $\mathrm{p}<0.01$ compared with the indicated group.

\section{KARGER}


the cytochrome c oxidase(COX) and mitochondrial ATP synthase activities in different groups. The results demonstrated that the OGD/R induced inhibition of COX and mitochondrial ATP synthase activities compared with NC group. Overexpression of HSPB8 significantly improved both COX activity and ATP synthase activity, when compared to cells transfected with control vector (Fig. 7C-7D). Overall, these data provided supporting evidence for the role of HSPB8 in protecting against OGD/R-induced mitochondrial dysfunction.

\section{Mitophagy suppression compromised the neuroprotection of HSPB8}

To clarify the contribution of mitophagy to the protective role of HSPB8 against OGD/R insult, mitophagy was blocked with the autophagy inhibitor CQ. The inhibitory effect of CQ on mitophagy was confirmed by western blot detection of TOMM20 and COX4I1 as shown in Fig. 3. Cell apoptotic rate was assessed using flow cytometry analysis. Cell apoptosis increased significantly in the group of CQ administration plus OGD/R compared with the pure OGD/R group, which suggested a beneficial role of mitophagy in mouse N2a OGD/R model. Moreover, when compared with the HSPB8 overexpressing group, apoptotic cell death was significantly higher in the HSPB8 and CQ co-treated group after OGD/R (Fig. 8A-8B). We

Fig. 7. HSPB8 improved mitochondrial function after OGD/R. Mouse N2a cells were subjected to $4 \mathrm{~h}$ OGD and then treated by $12 \mathrm{~h}$ reperfusion $\mathrm{A}$ caspase 3 , caspase 9and caspase 8 protein levels were determined by western blot analysis with HSPB8-myc or control vector transfection. B The statistical analysis of caspase protein levels. C-D. Cytochrome c oxidase and mitochondrial ATP synthase activities were normalized relative to the control. All data are representative of at least three

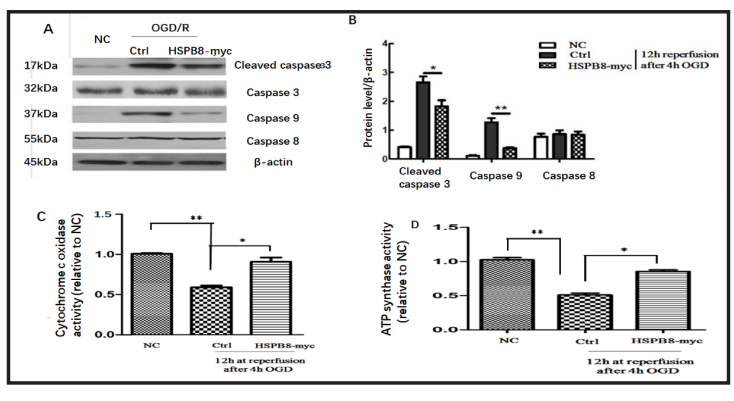
independent experiments. Experiments were repeated three times.Values are expressed as mean \pm SEM. * $\mathrm{p}<0.05$ compared to indicated group. $* * \mathrm{p}<0.01$ compared to indicated group.

Fig. 8. Mitophagy inhibition blocks HSPB8-mediated neuroprotection. Inhibitors of autophagy, $10 \mu \mathrm{mol} / \mathrm{L}$ CQ or $25 \mu \mathrm{mol} / \mathrm{L}$ mdivi-1, were applied at the onset of reperfusion. A.Cell apoptosis was assessed by flow cytometric analysis. The $y$-axis represents PI and the $\mathrm{x}$-axis represents annexinV/FITC staining.The cell distributions in flow cytometric histograms are as follow: Lower left quadrants, intact cells. Lower right quadrants, early apoptotic cells. Upper left quadrants, necrotic cells. Upper right quadrants, nonviable late apoptotic cells. The bars depicting the apoptosis rate of different groups. B. Quantitative analysis of apoptotic rate. Total percentage

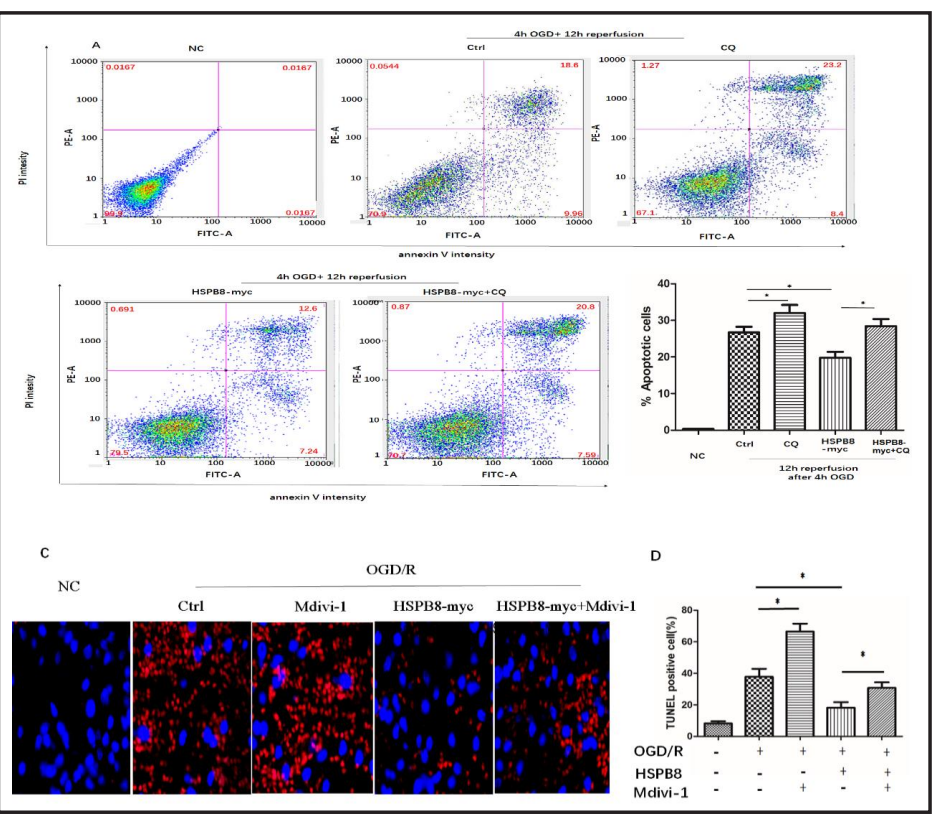
value of apoptotic cells included both early- and late-staged apoptosis. C. Photomicrographs show TUNEL/DAPI staining in separated groups. D. Bar graphs indicate quantitative analysis of TUNEL-positive cells. Experiments were repeated three times. Values are expressed as mean \pm SEM. $*$ p $<0.05$ compared to indicated group. 
further blocked mitophagy by mdivi-1. Mdivi-1 is DNM1L (dynamin 1-like) inhibitor, which blocks mitochondrial fission and thus makes it difficult for the mitochondria to be engulfed by phagophores [14]. Cell apoptosis was assessed by TUNEL at $12 \mathrm{~h}$ reperfusion after OGD. The results showed that mdivi-1 impaired the antiapoptotic effect of HSPB8. Taken together, these data offered a support for the hypothesis that HSPB8 mediated neuroprotection against OGD/R through, at least partly, enhancing mitophagy.

\section{Discussion}

In the present research, we identified that HSPB8 was constitutively upregulated after cerebral I/R injury in vivo and in vitro, implying that HSPB8 may be an important regulatory factor for neuronal survival during the process. Interestingly, we observed the increase in HSPB8 mRNA level earlier than at the protein level. It is well known that transient translational arrest is a stereotypical response to a variety of cell stresses including ischemia [15]. Subsequent resumption of translation resulted in HSP mRNA being selectively translated into HSPs [16]. Mizzen and Welch observed that after heat shock, cells allowed a $4 \mathrm{~h}$ recovery period to require some degree of translational tolerance [17], a time frame similar with our observations. In addition, HSPB8 gene expression in N2a cells at $4 \mathrm{~h}$ and $12 \mathrm{~h}$ perfusion after OGD were significantly higher than that in cells subjected 4h OGD without reperfusion. The finding suggested reperfusion rather than ischemia robustly induced HSPB8.

It is of particular interest that BAG3, a well-known trigger for autophagy, acts as an obligate partner of HSPB8 in several cell lines. HSPB8 complexes with BAG3 [18], efficiently facilitating autophagic removal of specific substrates, such as $\alpha$-synuclein, mutant SOD1 and aberrantly long polyglutamine tracts. In this way, HSPB8 counteracts neurotoxic events in many neurodegenerative diseases including Parkinson disease(PD) [19], Amyotrophic lateral sclerosis(ALS) [8] and spinal and bulbar muscular atrophy [20]. In our study, the results showed that concurrently with the upregulation of HSPB8 expression induced by brain I/R, the LC3-I to LC3-II turnover increased and p62 decreased, which indicated autophagy activation. Interestingly, the protein levels of two mitochondria markers TOMM20 and COX411 also reduced accompanied with HSPB8 upregulation at different reperfusion time points, suggesting the activation of mitophagy in our focal cerebral ischemic model. The results in our present investigation that HSPB8 upregulation and mitophagy activation were significantly and positively correlated, combined the fact that HSPB8 plays a significant role in autophagy, prompted us to investigate whether HSPB8 may trigger mitophagy in brain I/R condition.

In this study, we investigated the role of HSPB8 overexpression in regulating mitophagy in OGD/R-stimulated N2a cells in vitro. After OGD/R, in HSPB8 overexpressing N2a cells, LC3-II turnover was markedly up-regulated and p62 was significantly decreased, suggesting that autophagic flux was robustly reinforced. The mitochondrial markers TOMM20 and Cox4l1 were noticeably downregulated. The possible contribution of reduced mitochondrial biogenesis to TOMM20 and COX411 protein loss was excluded by RT-PCR. Lysosome inhibition reversed the mitochondrial loss confirmed that HSPB8-induced autophagic pathway was involved in mitochondria clearance. Additionally, OGD/R heavily modified the intracellular distribution of LC3, from a diffuse staining to the typical punctate staining colocalized with mitochondria. Activation of mitophagy by OGD/R suggested that mouse N2a cell had a self-repairing capability to clear dysfunctional mitochondria specifically by mitophagy after ischemic stress. However, when subjected to the severe injury, mitochondrial quality control system might become overloaded, thus resulting in irreversible and permanent damage. Specifically, HSPB8 overexpression further elevated the level of LC3-II conversion and decreased the expressions of Cox411 and TOMM20 to a significant extent. Increased overlapping signals of mitochondria and autophagosomes in HSPB8 overexpressing cells validated that mitophagy was augmented by HSPB8. Moreover, the result that HSPB8 silence suppressed mitophagy further supported the role of HSPB8 in mitophagy process in mouse 
Fig. 9. The potential neuroprotective mechanism of HSPB8 against OGD/R injury. In our study, HSPB8 overexpression in mouse N2a cell lines was achieved by vector transfection. HSPB8 overexpression could enhance autophagic degradation of mitochondria after $12 \mathrm{~h}$ reperfusion post-OGD, evidenced by the data from westernblot and immunofluorescence. Conversely, siRNA successfully knocked down the HSPB8 expression in N2a cell, and reduced mitophagy was observed in siRNA HSPB8 transfected cells using western-

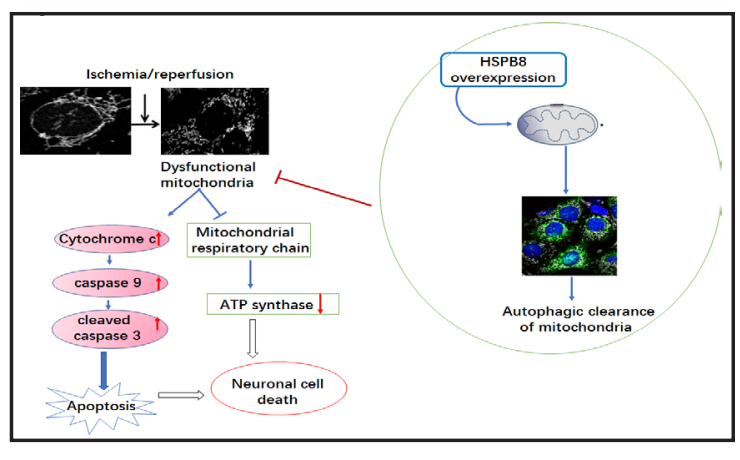
blot, immunofluorescence and TEM technologies.

To investigate the role of HSPB8 overexpression enhanced mitophagy in OGD/R injury, flow cytometric analysis was adopted to measure apoptotic rate. Moreover, western-blot was taken to assess mitochondriaassociated apoptosis and COX activity and ATP synthase activity assay were used to investigate the mitochondrial function. The results showed that HSPB8 overexpression significantly restored mitochondrial function and interfered with mitochondrial apoptotic pathway. Mitophagy inhibition by 3-MA or mdivi-1 blocked the protective effect. Therefore, HSPB8 overexpression is protective against OGD/R injury via enhancing mitophagy.

N2a cells after OGD/R.

The effects of mitophagy on cerebral ischemia brain injury have been controversial for years. Accumulation of damaged mitochondria has been observed following ischemia, which is probably a consequence of insufficient mitophagy [21]. Failure to remove dysfunctional mitochondria will inevitably result in activation of apoptotic signaling pathway, and thus the protection role of mitophagy has been proposed [22]. Nevertheless, mitophagy is not always protective, as excessive degradation of essential mitochondria will create an energy deficiency and result in cell death [23]. The enhancement of mitophagy by HSPB8 hence may represent either as a mechanism to recycle injured mitochondria and reduce damage or a process leading to cell demise. Therefore, it should be noted that whether increased mitophagic activity induced by HSPB8 is pathological or protective. In the present study, our data assign to HSPB8 a role in protecting mouse N2a cells from OGD/R-induced apoptosis evidenced by decreased apoptotic rate and reduced caspase-3 expression. As for ischemic injury, cumulative evidence suggests the involvement of both mitochondria-related and death receptor-mediated pathway in the pathogenesis of apoptotic cell death in ischemic lesions $[24,25,26]$. In consistent with the data from our previous report [27], we clearly showed that HSPB8 decreased caspase-9 expression without impacting on caspase-8 expression, implying HSPB8 interfered specifically with the mitochondria-dependent apoptotic pathway upon OGD/R insult.

Surprisingly, we found that HSPB8 overexpression significantly attenuated OGD/Relicited decreased activity of cytochrome c oxidase. Additionally, OGD/R decreased the ability of mitochondria to synthesize ATP, which was markedly improved by HSPB8 overexpression. It is seemingly contradictory that HSPB8 increased COX activity and decreased mitochondrial proteins. It is widely accepted that the catalytic activity of mammalian COX is regulated by binding of ATP to the N-terminus of subunit IV [28]. So far, two isoforms of COX subunit IV (IV-1 and IV-2) has been detected in yeast, tuna fish, and mammals. Under hypoxic conditions, the switch from IV-1 to IV-2 represents an adaptive response that optimizes COX activity [29]. Surprisingly, only a partial expression of COX isoform IV-2 seems sufficient to cause an abolition of the allosteric inhibition of COX activity [30]. Therefore, we speculated that besides inducing mitophagy, HSPB8 may also manipulate COX4 subunit switch to optimize efficiency of respiration in hypoxic cells. This presumption waits for further study in future.

To further verify the involvement of mitophagy in the protective role of HSPB8 against OGD/R injury, we inhibited mitophagy with CQ or mdivi-1. Here we showed that suppression of mitophagy either by CQ or mdivi-1 exacerbated OGD/R-associated cell apoptosis,

\section{KARGER}


indicating that mitophagy activation is an endogenous protective mechanism in response to ischemic injury rather than a cause of neuronal death. Moreover, blockade of mitophagy counteracted the antiapoptotic effect of HSPB8 in OGD/R-treated N2a cells, which provided a strong evidence that mitophagy is required for the protective effect of HSPB8.

In summary, the present study suggested that HSPB8 overexpression protected neuronal cells against $\mathrm{OGD} / \mathrm{R}$ injury by reinforcing mitophagy, which bring forth the notion that mitochondria are critical targets of HSPB8-induced autophagy (Fig. 9). Therefore, HSPB8 can be a promising neuroprotective agent for cerebral ischemia treatment.

\section{Acknowledgements}

This study was supported by the National Science Foundation of Hunan Province, China (2018JJ3749).

\section{Disclosure Statement}

The authors declare that there is no conflict of interests regarding the publication of this paper.

\section{References}

1 Carra S, Seguin SJ, Lambert H, Landry J: HspB8 chaperone activity toward poly(Q)-containing proteins depends on its association with Bag3, a stimulator of macroautophagy. J Biol Chem 2008;283:1437-1444.

-2 Depre C, Tomlinson JE, Kudej RK, Gaussin V, Thompson E, Kim SJ, Vatner DE, Topper JN, Vatner SF: Gene program for cardiac cell survival induced by transient ischemia in conscious pigs. Proc Natl Acad Sci USA 2001;98:9336-9341.

- 3 Depre C, Kim SJ, John AS, Huang Y, Rimoldi OE, Pepper JR, Dreyfus GD, Gaussin V, Pennell DJ, Vatner DE, Camici PG, Vatner SF: Program of cell survival underlying human and experimental hibernating myocardium. Circ Res 2004;95:433-440.

4 Depre C, Wang L, Sui X, Qiu H, Hong C, Hedhli N, Ginion A, Shah A, Pelat M, Bertrand L, Wagner T, Gaussin V, Vatner SF: H11 kinase prevents myocardial infarction by preemptive preconditioning of the heart. Circ Res 2006;98:280-288.

5 Chen L, Lizano P, Zhao X, Sui X, Dhar SK, Shen YT, Vatner DE, Vatner SF, Depre C: Preemptive conditioning of the swine heart by H11 kinase/Hsp22 provides cardiac protection through inducible nitric oxide synthase. Am J Physiol Heart Circ Physiol 2011;300:H1303-H1310.

6 Yang B, Zhang H, Mo X, Xiao H, Hu Z: HspB8 is neuroprotective during oxygen glucose deprivation and reperfusion. Curr Neurovasc Res 2015;12:63-72.

7 Jo HS, Kim DW, Shin MJ, Cho SB, Park JH, Lee CH, Yeo EJ, Choi YJ, Yeo HJ, Sohn EJ, Son O, Cho SW, Kim DS, Yu YH, Lee KW, Park J, Eum WS, Choi SY: Tat-HSP22 inhibits oxidative stress-induced hippocampal neuronal cell death by regulation of the mitochondrial pathway. Mol Brain 2017;10:1.

8 Crippa V, Sau D, Rusmini P, Boncoraglio A, Onesto E, Bolzoni E, Onesto E, Bolzoni E, Galbiati M, Fontana E, Marino M, Carra S, Bendotti C, De Biasi S, Poletti A: The small heat shock protein B8 (HspB8) promotes autophagic removal of misfolded proteins involved in amyotrophic lateral sclerosis (ALS). Hum Mol Genet 2010;19:3440-3456.

-9 Ganassi M, Mateju D, Bigi I, Mediani L, Poser I, Lee HO, Seguin SJ, Morelli FF, Vinet J, Leo G, Pansarasa O, Cereda C, Poletti A, Alberti S, Carra S: A Surveillance Function of the HSPB8-BAG3-HSP70 Chaperone Complex Ensures Stress Granule Integrity and Dynamism. Mol Cell 2016;63:796-810.

-10 Crippa V, D’Agostino VG, Cristofani R, Rusmini P, Cicardi ME, Messi E, Loffredo R, Pancher M, Piccolella M, Galbiati M, Meroni M, Cereda C, Carra S, Provenzani A, Poletti A: Transcriptional induction of the heat shock protein $B 8$ mediates the clearance of misfolded proteins responsible for motor neuron diseases. Sci Rep 2016;6:22827. 


\section{Cellular Physiology Cell Physiol Biochem 2018;48:1492-1504 \begin{tabular}{l|l} 
DOI: 10.1159/000492259 & $\begin{array}{l}\text { O 2018 The Author(s). Published by S. Karger AG, Basel } \\
\text { www.karger.com/cpb }\end{array}$
\end{tabular}}

Li et al.: Neuroprotective Role of HSPB8 Induced Mitophagy in OGD/R

11 Zhang X, Yan H, Yuan Y, Gao J, Shen Z, Cheng Y, Shen Y, Wang RR, Wang X, Hu WW, Wang G, Chen Z: Cerebral ischemia-reperfusion-induced autophagy protects against neuronal injury by mitochondrial clearance. Autophagy 2013;9:1321-1333.

12 DeGracia DJ, Jamison JT, Szymanski JJ, Lewis MK: Translation arrest and ribonomics in post-ischemic brain: Layers and layers of players. J Neurochem 2008;106:2288-2301.

13 Cherkasov V, Hofmann S, Druffel-Augustin S, Mogk A, Tyedmers J, Stoecklin G, Bukau B: Coordination of translational control and protein homeostasis during severe heat stress. Curr Biol 2013;23:2452-2462.

14 Mizzen LA, Welch WJ: Characterization of the thermotolerant cell. I. Effects on protein synthesis activity and the regulation of heat-shock protein 70 expression. J Cell Biol 1988;106:1105-1116.

15 Behl C: BAG3 and friends: Co-chaperones in selective autophagy during aging and disease. Autophagy 2011;7:795-798.

16 Bruinsma IB, Bruggink KA, Kinast K, Versleijen AA, Segers-Nolten IM, Subramaniam V, Kuiperij HB, Boelens W, de Waal RM, Verbeek MM: Inhibition of $\alpha$-synuclein aggregation by small heat shock proteins. Proteins Struct Funct Bioinforma 2011;79:2956-2967.

17 Carra S, Sivilotti M, Zobel AT, Lambert H, Landry J: HspB8, a small heat shock protein mutated in human neuromuscular disorders, has in vivo chaperone activity in cultured cells. Hum Mol Genet 2005;14:16591669.

18 Liu C, Gao Y, Barrett J, Hu B: Autophagy and protein aggregation after brain ischemia. J Neurochem 2010;115:68-78.

19 Kubli DA, Gustafsson ÅB: Mitochondria and mitophagy: The yin and yang of cell death control. Circ Res 2012;11:1208-1221.

20 Chen H, Chan DC: Mitochondrial dynamics-fusion, fission, movement, and mitophagy-in neurodegenerative diseases. Hum Mol Genet 2009;18:R169-176.

21 Sugawara T, Noshita N, Lewen A, Gasche Y, Ferrand-Drake M, Fujimura M, Morita-Fujimura Y, Chan PH: Overexpression of copper/zinc superoxide dismutase in transgenic rats protects vulnerable neurons against ischemic damage by blocking the mitochondrial pathway of caspase activation. J Neurosci 2002;22:209-217.

-22 Chen J, Nagayama T, Jin K, Stetler RA, Zhu RL, Graham SH, Simon RP: Induction of caspase-3-like protease may mediate delayed neuronal death in the hippocampus after transient cerebral ischemia. J Neurosci 1998;18:4914-4928.

23 Plesnila N, Zinkel S, Le DA, Amin-Hanjani S, Wu Y, Qiu J, Chiarugi A, Thomas SS, Kohane DS, Korsmeyer SJ, Moskowitz MA: BID mediates neuronal cell death after oxygen/ glucose deprivation and focal cerebral ischemia. Proc Natl Acad Sci U S A 2001;98:15318-15323.

24 Yang B, Zhang H, Mo X, Xiao H, Hu Z: HspB8 is neuroprotective during oxygen glucose deprivation and reperfusion. Curr Neurovasc Res 2015;12:63-72.

25 Pierron D, Wildman DE, Hüttemann M, Markondapatnaikuni GC, Aras S, Grossman LI: Cytochrome c oxidase: Evolution of control via nuclear subunit addition. Biochim Biophys Acta 2012;1817:590-597.

-26 Solaini G, Baracca A, Lenaz G, Sgarbi G: Hypoxia and mitochondrial oxidative metabolism. Biochim Biophys Acta 2010;1797:1171-1177.

-27 Horvat S, Beyer C, Arnold S: Effect of hypoxia on the transcription pattern of subunit isoforms and the kinetics of cytochrome c oxidase in cortical astrocytes and cerebellar neurons. J Neurochem 2006;99:937951.

28 Fan YY, Hu WW, Dai HB, Zhang JX, Zhang LY, He P, Shen Y, Ohtsu H, Wei EQ, Chen Z: Activation of the central histaminergic system is involved in hypoxia-induced stroke tolerance in adult mice. J Cereb Blood Flow Metab 2011;31:305-314.

-29 Hurtado O, Lizasoain I, Fernández-Tomé P, Alvarez-Barrientos A, Leza JC, Lorenzo P, Moro MA: TACE/ ADAM17-TNF-alpha pathway in rat cortical cultures after exposure to oxygen-glucose deprivation or glutamate. J Cereb Blood Flow Metab 2002;22:576-585.

-30 Back SA, Craig A, Kayton RJ, Luo NL, Meshul CK, Allcock N, Fern R: Hypoxia-ischemia preferentially triggers glutamate depletion from oligodendroglia and axons in perinatal cerebral white matter. J Cereb blood flow Metab 2007;27:334-347. 